\title{
Distribution of residual deformation effects in shell tubes from ferritic-martensitic steels
}

\author{
Yuriy Perlovich ${ }^{\mathrm{a}}$, Margarita Isaenkova ${ }^{\mathrm{b}}$, Evgeniy Zharikov, Olga Krymskaya \\ National Research Nuclear University (MEPhl), Moscow, Russia \\ ayuperl@mail.ru, bisamarg@mail.ru
}

Keywords: steel tube, texture, X-ray line profile, generalized pole figure, substructure inhomogeneity,

\begin{abstract}
The X-ray method of Generalized Pole Figures was used for the study of residual deformation effects in steel shell tubes for high-temperature atomic reactor. Ferritic-martensitic steel contained dispersed strengthening oxide particles and their behavior under technological treatment was of essential practical interest. The used procedure estimates residual deformation effects in grains with different orientations and depicts distributions of elastic lattice distortion and interplanar spacings. Oxide particles, invisible for X-rays, redistribute within the tube wall, so that strain hardening at its outward side increases.
\end{abstract}

\section{Introduction}

Ferritic-martensitic steels presently are most prospective materials for production of shells and covers for high-temperature nuclear reactors. This is conditioned by their small induced activity, low vacancy swelling, high stability relative to high-temperature embrittlement. Ferritic-martensitic steels with oxide dispersed strengthening additions (ODS) are of the special interest since, besides indicated properties, they show also stability to high-temperature creep [1]. Study of structure features as applied to these steels is of important practical and fundamental significance. By manufacture of concrete products from these steels a structure inhomogeneity inevitably arises, and its analysis promotes understanding of mechanisms, responsible for properties, which distinguish these steels from other reactor materials.

\section{Studied samples and methods of investigation}

The given paper is devoted to the study of tubes from ferritic-martensitic ODS steel EP-450, containing 0,35 mass. $\%$ of yttrium oxide $\mathrm{Y}_{2} \mathrm{O}_{3}$ particles up to $20 \mathrm{~nm}$ in size. Methods of contemporary X-ray diffractometry were used. Tubes of $13,0 \mathrm{~mm}$ in diameter with wall thickness $1,0 \mathrm{~mm}$ had different structure conditions - after cold rolling and after heat treatment, accompanied with recrystallization.

X-ray studies included texture analysis by methods of direct and reverse pole figures [2-3] and analysis of substructure inhomogeneity by the method of generalized pole figures (GPF) [4]. Registration of diffraction spectra was conducted for studied samples at their outward and inward surfaces in order to reveal effects, conditioned by operation of macrostress.

The X-ray diffractometer Bruker D8 Discover with a position-sensitive detector was used. The complex of computer programs was used by treatment of measured X-ray data, including large number of experimental results. These programs were developed by authors in parallel with the GPF method.

The GPF method was applied in the following way. For structure condition characterization of grains with different crystallographic orientations X-ray lines are registered by different positions of the studied sample in the process of its texture measurement. We use the selectivity of X-ray diffraction, due to which the X-ray line gives information only concerning structure features of grains, being in the reflecting condition. By mathematical treatment of X-ray line profiles we 
determine values of physical half-width and angular position, connected with substructure parameters of grains, measured along normals to reflecting planes.

\section{Experimental results and discussion}

In the given case application of the GPF method allows to estimate the strengthening caused by disperse oxide particles, which were added in steel. Features of plastic deformation mechanisms in steel in the presence of strengthening disperse phase can be revealed as well. Changes of substructure condition of matrix in the presence of disperse strengthening particles helps to estimate indirectly the influence of additions on the steel structure, though direct observation of the oxide phase by diffraction pattern proves to be impossible.

Since manufacture of tubes is connected with large plastic deformations, tube material proves to be strongly textured and, as in any metal material with a developed deformation texture, significant substructure inhomogeneity arises in it. This inhomogeneity consists in sharp differences of strain hardening and tendencies to following recrystallization in different grains depending on their place in the tube texture. At that, additions of disperse strengthening particles into steel can result in redistribution of strain hardening, typical for the initial matrix, which did not contain these particles. Only the GPF method allows to split information on the structure of material into data, relating to grains of different texture components with orientations, distanced from texture maxima and minima by different angular intervals.

As an illustration of possibilities of the used approach to study tubes from ODS steel, the following results are considered. Fig. 1 shows (a) the direct pole figure $\operatorname{PF}\{001\}$ for inward side of tube wall, (b) the generalized pole figure GPF $\beta_{002}$, showing the distribution of true half-width for $\mathrm{X}$-ray line for the same sample, and (c) the diagram of correlation between GPF $\beta_{002}$ and PF $\{001\}$, where abscissa of each point $(\varphi, \psi)$ in the correlation diagram is equal to pole density in this point of $\mathrm{PF}\{001\}$, while ordinate is equal to angular half-width $\beta_{002}$ in the same point of GPF $\beta_{002}$.

The Generalized Pole Figures method consists in successive diffractometric registration of the same X-ray line (hkl) profile for grains of all orientations, presented in the studied sample, in the course of its texture measurement. According to the X-ray diffraction theory [5], each profile characterizes a condition of the crystalline lattice in reflecting grains along axes $<\mathrm{hkl}\rangle$. Measured parameters of X-ray lines, i.e. intensity $I$, angular position $2 \theta$, angular half-width $\beta$, are connected with grain substructure parameters, in particular with lattice distortion $\Delta d / d$ and coherent domain size $D$. Then GPF $(\Delta d / d)$ and GPF $D$ give the most complete X-ray description of the substructure, formed in the studied textured sample.

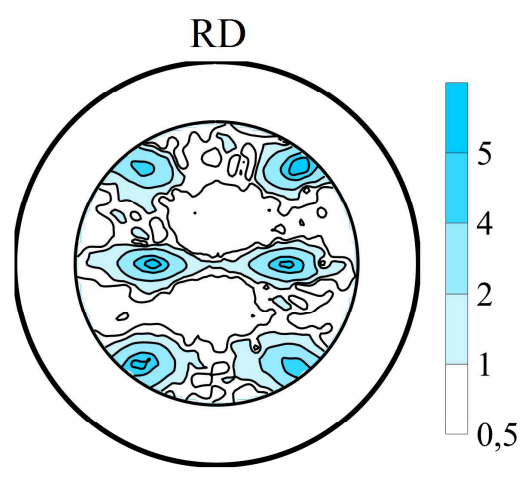

a

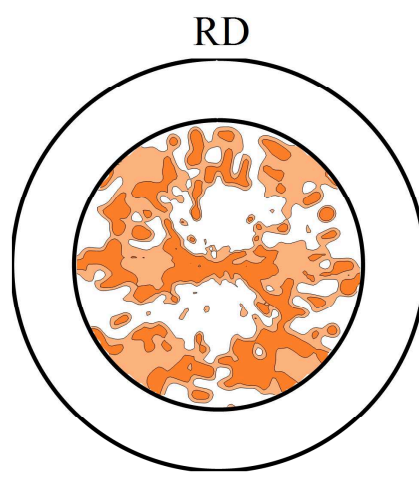

b

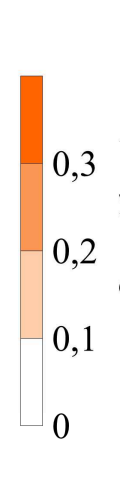
(1)

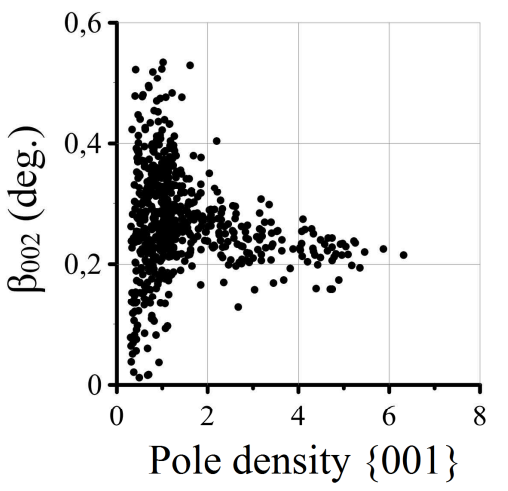

c

Fig. 1. Substructure inhomogeneity of tube from steel EP-450 ODS, cold rolling, inward side of tube wall: (a) PF $\{001\}$; (b) GPF $\beta_{002}$; (c) diagram of correlation between GPF $\beta_{002}$ and $P F\{001\}$. 
Analogous pole figures and diagram, constructed for outward side of tube wall in Fig. 2, have similar appearances, but are essentially more scattered. At that, the mean level of values $\beta_{002}$ for outward side of the tube wall significantly exceeds that for its inward side.

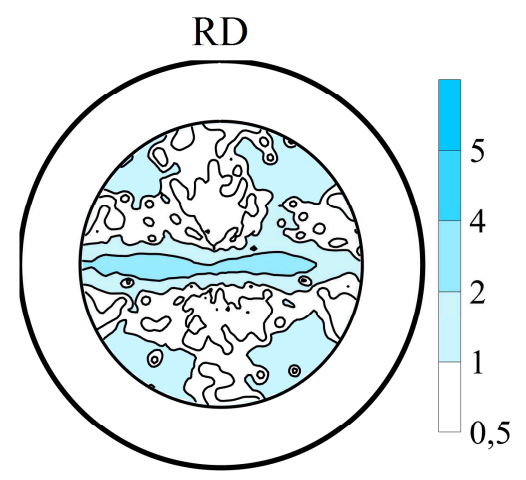

a

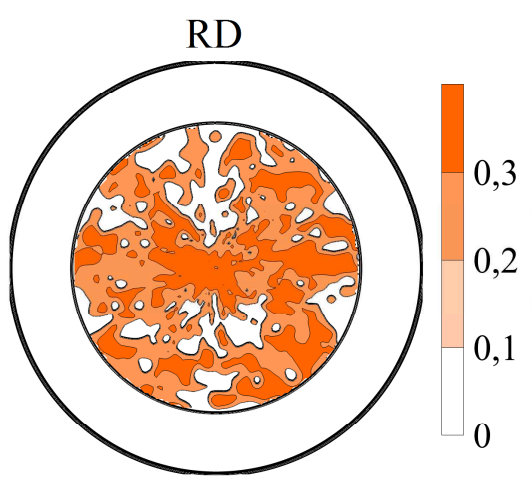

b

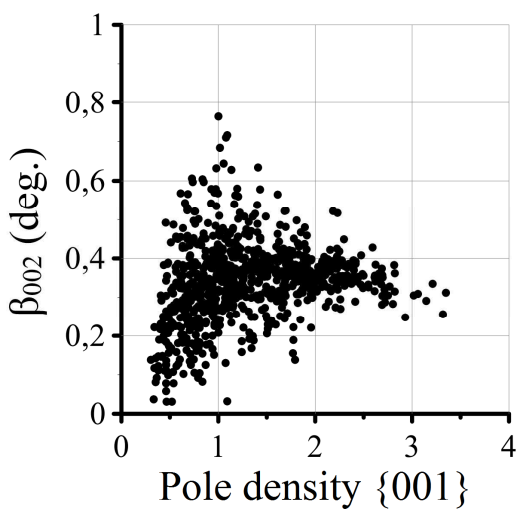

c

Fig. 2. Substructure inhomogeneity of tube from steel EP-450 ODS, cold rolling, outward side of tube wall: (a) PF $\{001\}$; (b) GPF $\beta_{002}$; (c) diagram of correlation between GPF $\beta_{002}$ and $\operatorname{PF}\{001\}$.

The character of texture, formed at different regions of sample and observed by direct pole figures, also confirms features of layer-by-layer distribution of ODS additions in the tube wall. Near inward surface of the rolled sample the texture noticeably sharpens (compare Figs. 1-a and 2-a). This fact testifies that crystallographic slip becomes more regular under conditions of lesser lattice distortions. In the given case this distortion is connected with disperse oxide particles, distributed in the tube volume.

Additional scattering of texture maxima in Fig. 2-a, as compared with that in Fig. 1-a, indicates that the attainment of texture maxima by grains at the outward side of tube happens more difficult, than by grains at its inward side. The reason of this difference consists in more difficult slip in the presence of grater quantity of ODS particles. More extensive regions of scattering in GPF $\beta_{002}$ (Fig. 2-b) confirm the higher strengthening of most grains at the outward side of tube.

Histograms of X-ray line true (002) half-width distributions are shown in Fig. 3. They characterize conditions of $\alpha$-Fe crystalline lattice along axes $<001>$ and $<011>$ in GPF $\beta_{002}$ and GPF $\beta_{011}$ for outward and inward layers of tube.

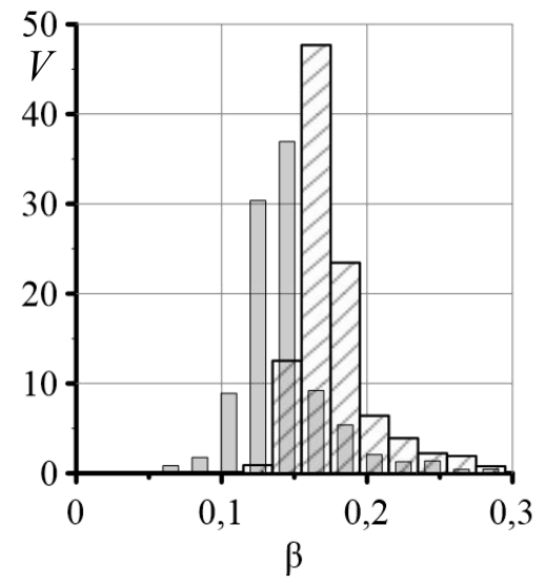

a

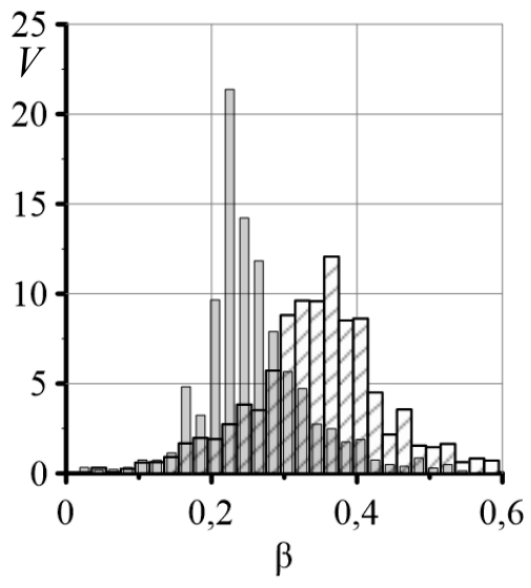

b

\section{日}

outward layer

inward layer

Fig. 3. Histograms of distributions of volume fractions of grains, characterizing by different distortion of crystalline lattice along axes $<110>$ (a) and $<100>$ (b),

for outward and inward tube layers. 
Since the content of disperse-strengthening particles in steel is too small in order to be directly registered, we appreciate their influence on the steel substructure by accompanying effects and in the given case - by additional broadening of X-ray lines for outward layers of tube. Therefore we conclude, that in the process of tube rolling disperse strengthening particles redistribute in such a manner, that in outward layers of tube there are more oxide particles, than in inward layers. Measurements of lattice parameter confirm, that tensile macrostresses operate in outward layers of tube. Thus, X-ray data, obtained by different methods, agree with each other.

\section{Conclusions}

(1) Structure of deformed ferritic-martensitic steels includes wide spectrum of different substructure conditions.

(2) The most effective criterion for systematization of substructure inhomogeneity in textured metal materials is crystallographic orientation of grains.

(3) Though the content of disperse-strengthening particles in steel tubes is less, than the sensitivity limit of diffractometric measurements, one can successfully estimate the behavior of oxide additions by their influence on the character of diffraction pattern from $\alpha$-Fe matrix.

(4) As a result of cold rolling significant substructure inhomogeneity develops in tube; at that strain hardening in layers, adjacent to the outward side of tube wall, is essentially higher, than in layers, adjacent to its inward side.

(5) Increased strain hardening of the matrix near outward side of tube wall indicates the redistribution of disperse-strengthening particles in the process of extrusion and cold rolling, promoted by arising there tensile tangential macrostresses.

\section{References}

[1] A. Steckmeyer, M. Praud, B. Fournier et al. - Journal of Nuclear Materials, 2010, v. 405, p. 95100.

[2] M. Borodkina, E. Spector, X-ray texture analysis of metals and alloys, Metallurgy (1981).

[3] Yu.A. Perlovich, M.G. Isaenkova, S.N. Kaplii, Rapid Method of Mapping the Inverse Pole Figures, Industrial Laboratory, vol. 79, № 05 (2013) 27-30.

[4] Yu. Perlovich, H.-J. Bunge, M. Isaenkova - Textures \& Microstructures, 1997, v. 29, p. 241266.

[5] Warren B.E. X-ray diffraction. - Addison-Wesley Publishing Company, Inc., Reading, Massachussetts, 1969. - $381 \mathrm{p}$. 